\title{
Multi-Level and Ecological Models of Developmental Coordination Disorder
}

\author{
David Sugden
}

Published online: 15 March 2014

(C) Springer International Publishing AG 2014

\begin{abstract}
In order to explain the difficulties experienced by children with Developmental Coordination Disorder (DCD), and to indicate ways forward for intervention, two overlapping ecological models are described. The first examines a bio-ecological model that explains and promotes the participation of children with DCD into physical activity. This model involves a nested set of concepts of the environment moving from the wider social environmental and policy context to the narrower child-person interaction. The second, a model based upon the transaction of perception and action, is one that explains the performance and learning of the child once they are in a participatory context, drawing upon the concept of movement taking place in a meaningful environment that is rich enough to invite appropriate action. Both models offer optimism, based upon the principle that the environment can be changed to one that is more meaningful to the child, allowing maximum participation and skill learning.
\end{abstract}

Keywords Developmental Coordination Disorder . Ecological models $\cdot$ Physical activity participation $\cdot$ Physical activity learning/performance $\cdot$ Perception action .

Organism-environment

\section{Introduction: Ecological Models}

Children with Developmental Coordination Disorder (DCD) show a wide variety of characteristics in the motor domain and in other areas such as cognitive, social and emotional. Understanding these in children with DCD, as in all children, necessitates different levels and modes of explanations. These explanations not only apply to characteristics, but also have

D. Sugden $(\square)$

School of Education, University of Leeds, Leeds LS2 9JT, UK

e-mail: d.a.sugden@leeds.ac.uk implications for assessment and intervention. Children with DCD are defined by their acquisition and execution of coordinated motor skills being substantially below expectations given chronological age and opportunity. This difficulty significantly and persistently interferes with academic activities or activities of daily living, occurs within the early developmental period, and is not better explained by other factors such as intellectual difficulties or a medical condition such as cerebral palsy $[1 \bullet \bullet]$. Despite these difficulties, children with DCD do have strengths and the argument made here is that all strengths and weaknesses are best explained by models, which can be biological, cognitive, environmental or a combination thereof. These models can be used for all individuals, not just children, whether their behaviour is typical or atypical.

Two variables are important when considering the lives of children with DCD. The first of these is participation and the second is the twin concepts of performance and learning. Participation is not a unitary variable but involves different parts, including how easily accessible appropriate contexts are made; ensuring that real life situations are personally and socially meaningful for the child, and how positive an influence they have on the child's life as viewed by all interested parties. Emotional engagement in any activity is also a key feature of appropriate participation, and can be measured by time on task and verbal reports. Reports detail how participation involves a range of activities in different contexts, such as household work in the home, community based leisure activities and full participation in school activities [2]. This line of thought follows directly the WHO model that clearly separates functioning from ability or disability, and has a strong emphasis on participation, with factors outside of the child being the major influences [3]. The second variable involves the twin concepts of performance and learning, with an emphasis on those factors affecting the task performance itself and the change in capacity over time. Once the child is in a situation where participation is possible with meaningful engagement, 
and having learnt and performed the task, an examination of the models that explain skilled motor performance assists in understanding the child's difficulties. The influences that affect the performance and learning are different from those relating to performance, yet there are commonalities.

In order to explain and account for both participation and learning and performance two complementary but different theoretical bases are presented. They both invoke the term 'ecological', take different meanings of the concept, yet overlap in some of their fundamental principles and have similar implications for the study of DCD. The first of these models addresses participation and how this can be explained in children with DCD. The second alludes to performance and learning, addressing the motor core of difficulties in children with DCD. A fuller version of many of the following positions can be found in a recent journal Special Edition [4••].

\section{Bronfenbrenner and Ecology}

A famous ecological model is the one proposed in many publications by Bronfenbrenner $[5,6]$, where human development involves more progressively complex reciprocal interactions between the organism and the immediate environment, which can change the organism. The ecological environment is conceived as a set of nested structures inside each other like a group of Russian dolls. At the centre of this structure is the micro-system, which is the immediate environment such as the family, school, peers and workplace. In this, as an example, the mother-infant interaction is viewed not only as important, but also able to buffer some deleterious effects of the wider environment. The microsystem is set within the meso-system, which is akin to a set of microsystems working together in two or more settings. This in turn is set within the exo-system, which also involves two or more settings, but although the influences are on the child, it does not always include the developing person. Finally, at the outside there is the macro-system that operates on the pattern of micro, meso and exo-systems, and would include such variables as belief systems, life style, social and psychological features, going beyond mere labels of class and culture. Cutting across all these systems is the chrono-system that looks at changes as development takes place; it is not simply a product of time but also the changes that occur with time in both the person and the environment. It is a bio-ecological model, and particular mention is made of the defining dimensions of process, time, person and context, with the proximal processes being a primary mechanism for producing development. The proximal processes involve the child interacting with the environmental context on a regular basis over a protracted period of time, involving activities with significant others, such as playing with a parent and reading together, which help children come to understand their world and find their place in it.
The model provides a sound theoretical framework towards an understanding of the developmental process of children with DCD, with a central message that any difficulties a child may have are a function of the resources of the child and the various environmental influences. These environmental influences range from wide societal pressures to ones specifically dealing with the child engaging in a physical activity.

\section{Turvey and Ecology}

A different, yet complementary model is provided in numerous publications by Turvey $[7 \cdot, 8 \bullet \cdot]$. These follow the ideas of Gibson [9] and his work on the links between perception and action. Here, the focus of psychology is how any organism manages, to some degree, the environmental niche in which it is situated. Their work would probably fit at the centre of the Bronfenbrenner model, in the micro system and proximal processes, with the child directly interacting and moving in the immediate environment.

The theoretical base from which Turvey and others work questions the traditional view of perception being indirect, with the person having to construct meaning from the world, but is present in the complex transaction the child encounters with their environment. In particular, movement takes place in an environmental niche where the structure of the environment is in itself meaningful, through features they label affordances that invite appropriate action. The central concept of affordances involves what the environment offers to an organism to enable appropriate action. When a park bench is present, to a toddler it offers an opportunity to cruise around while holding on; for a three year old, a place to crawl under; for a bird, a landing place; for an adolescent, something to jump on, and for an adult, somewhere to sit. These are the environmental niches for an organism; they do not have to be constructed, they are already there and powerful enough to invite action. This view of ecology is directly concerned with the action of the child once participation is established.

\section{Commonalities}

These two approaches to ecological models started with different aims, one being to explain development, the other to explain skilful movement. Bronfenbrenner was aiming to provide a framework from which development could be understood by elaborating the different ecological systems that impinge on a child's life. Turvey and others were aiming to explain how perception and action are linked to produce the skilful movements we see every day.

The two bodies of research have approached different problems but they do share commonalities. Both of them either directly or tacitly reject the notion that the child can be studied in isolation from the context in which action takes place. Bronfenbrenner shows how the different ecologies in a 
child's life are powerful influences on outcomes. These environments are nested; from the tight one-to-one proximal influences in the microsystem; to the wider macro-system encompassing culture, social systems, attitudes and policies. Turvey and others have shown how the brain is not the sole determining factor in skilful movement, with other major influencing variables being interconnections between perception and action. This implies that the unit of analysis of movement is not simply the child but encompasses the context within which movement is performed, and the manner in which it is presented. When these are applied to children with $\mathrm{DCD}$, the contribution that Bronfenbrenner's model makes is to explore the environment to show how it can influence participation. Participation is essential for skill learning, with the amount of time devoted to appropriate practice being an important influencing variable to the final outcome. This is a logical generalisation and has support from work on overlearning of motor skills and expert performance and, although there have been a few exceptions to this statement, appropriate practice is a strong driver, and for this to occur participation is required $[10,11]$. By examining the context in which participation can be encouraged, Bronfenbrenner's model can be a theoretical framework from which therapists can work. Turvey's ideas on the other hand are more direct, in that they can be used to examine how performance and learning occur. By examining the environmental niche and accompanying invariants (i.e. those characteristics in the environment that remain unchanging), this work can influence performance and learning.

\section{Participation}

Participation involves appropriate engagement in the typical activities of the home, school and community and includes meaningful, positive and rewarding experiences for the child. When development and movement is typical, participation is often taken for granted, but with atypical development participation is often diminished. This could be because of a perceived lack of skill on the part of the child, or because the environment has not been structured enough to invite the child to participate. For any child, participation should provide a sense of well-being and self-fulfilment, with social interaction providing the opportunity to increase social skills and selfesteem. In addition, participation is absolutely necessary for learning to take place, and an increase in the amount of appropriate practice can only come about as a result of increased participation [12•]. Participation is not simply 'joining in', but should be real life situations that are meaningful, taking into account the views of the child, parent and significant others [13]. Work by Grandlund and colleagues in Sweden with their Child Health Intervention Learning and Development centre (CHILD) provides a good example, looking at participation and intervention in every day functioning for children with special educational needs.

It is well known that children with DCD participate less than their typically developing peers, therefore widening the gap between them and children without DCD. They participate less in organised sports and in free play activities. This was taken a stage further by examining whether this gap widens over time [14*0]. The study was conducted with children 9 to 11 years of age and participation was assessed by a questionnaire over three years on five occasions. The interesting results showed that the activity deficit persisted over the three-year period and that there was also a gender difference. In boys the activity gap diminished, whereas in girls it increased. This difference has important implications for future work on how the environment is structured or engineered to facilitate participation for both sexes. The relationship between coherence, hope, effort and participation in children with DCD was examined in a similar manner [15••]. The authors use coherence as a generic term and allencompassing concept reflecting predictability, flexibility and the application of a person's resources commensurate with the context. Hope is the perceived ability to construct and use pathways to achieve a goal, together the use of effort to engage the mental energy to start and persevere. The results showed children with DCD enjoyed participation less and their parents were less satisfied with the outcomes. The children with DCD had lower performance, lower sense of coherence and effort than their peers. A slightly different set of conditions were found by using questionnaires and accelerometers, with the result again that children with DCD not only had lower physical activity but also lower physical activity cognitions in relation to their activity and performance (i.e. metacognition), although this could be mediated by the approval of influential people in their lives [16••]. Thus we find that although deficits are found in participation and associated variables in children with DCD, they can be helped.

Much work has been completed on the physical activity participation of children with DCD, but only recently has there been a concerted effort to examine participation in all social life contexts, including school, home and community. This was taken up in an investigation that identified two groups of children with DCD: one with what they label as mixed Dyspraxia, that is Childhood Apraxia of Speech (CAS) and Developmental Dyspraxia (DD); and one solely with DD. These were both compared to typically developing children (TD). Both groups (DD, CAS) showed participation deficits compared to TD children. Factors such as differences in social participation, organizational skills, and processing skills leading to difficulties in engaging in curricula subjects, were all affected. The deficits related to communication, interpersonal relationships education and recreation, showing that overall lifestyle was affected. While this study was not definitive in its inference of causality, the authors did recommend the need for 
improved contacts both inside and outside the family, giving more time for tasks, adapting the work environment, and interactions with significant others [17••].

A review of participation in groups of children with more global neurodevelopmental disorders produced a number of common themes that captured the association between participation in leisure activities and quality of life in children with neurodevelopmental disorders [18•]. These themes involve:

- Active physical leisure participation being important for physical well-being.

- Leisure participation being associated with an increased sense of self perception, self-esteem, self-competence and also with being respected and treated fairly.

- Leisure participation leading to greater happiness and enjoyment in life.

- Leisure participation being important for social wellbeing, spending more time with peers and developing friendships.

- Leisure participation in various types of aerobic activities was found to be beneficial for cognition.

- Children choosing their own leisure preferences led to the children feeling better about themselves.

Negative aspects were also found in participation but again they were mainly environmental constraints such as equipment and surroundings not fully adapted, influence of more capable peers and financial constraints.

From this selection of recent studies two overriding factors stand out. The first is that children with neurodevelopmental disorders, or more specifically DCD, do not participate in leisure and other physical activities as much as their typically developing peers; they do not enjoy participation as much; their competence does not improve, as a result of lack of participation, and there are numerous spin-off deficits such as poorer peer relations, lower self-esteem and self-worth [19॰]. The second point is that these can be remedied to a certain degree by altering the participation variables. The Dahan-Oliel paper [18 $]$ shows the impact of leisure participation in a wider group of developmental disorders than simply DCD but it does not stretch science too far to infer that similar results would be found in children with DCD. The evidence for the lack of participation in children with DCD and its effects is overwhelming. Work invoking ecological models has been taken into the world of DCD and therapy with Ecological Intervention [20], and family centered function approaches [21], and appears to be the logical way forward to increase participation and impact, not just motor skills, but also social, emotional and cognitive variables in children. Participation provides the context within which children can perform, learn and find engagement; in interviews with teenagers with DCD, for example, it was noted that they wanted to be physically active and that there were many activities that they did enjoy [22].
Progress in participation can be made in small steps by different individuals. At school more reasonable adjustments can be made in the classroom, on playing fields, science laboratories and gymnasia. It has to be more than simply providing opportunities; it has to be positive, with the school environment making small changes in a number of areas that all serve to invite and promote, encourage and indeed cajole the child into participation. Progress is built up from the first principles of entitlement and not as an addition to the school's policies. This would start from the principle that all children have a right to equal opportunity and equal access; that any reasonable adjustments to the school context be provided in order for total inclusion to become a reality, with participation forming a crucial part. Such adjustments as adapting the materials for ease of manual skills; giving choice to a range of activities; selection of supportive individuals for group work; a no-option positive attitude from members of staff and other children should be common place. The same is true of the larger community with built-in access for all. In sports centers for example, this should not just be for disabled sports, but movement activities for all children, with different abilities, strengths and weaknesses. Can sports officers in clubs and centers go out and seek children with difficulties rather than wait for them to come in? How can they show the community that they want these children, that they will add to the kudos and popularity of the Centre and that their contribution will be valued as much as with any other child? In the home how can the family build participation into the child's life, with activities that are expected of them every day?

\section{Performance and Learning}

If we move to the center of the Bronfenbrenner model (the microsystem), the work of perception-action scientists takes us to a more fundamental level of performance and learning by describing and explaining how perception and action are inextricably linked in the performance and learning of skills.

The basic unit of analysis in these studies is not the child's resources but the childs resources set in the environmental context and how the action is displayed. This triangular transaction has been utilized many times but at a fundamental level is very simple $[23,24]$. It simply states that the outcome of a child's actions are the result of the transaction of these three components. The wider environmental context is often shown through the Bronfenbrenner model while the perception action model makes inroads into the performance and learning of a child. A link with the Bronfenbrenner model can be made by proposing that the ecological model of Turvey and others would lie somewhere in the processes of the microsystem of Bronfenbrenner. 
In a series of studies, the perception-action link in children with DCD was examined through manipulation of the support surfaces in a reaching and grasping task $[25,26 \bullet, 27 \bullet]$. The children with DCD were required to both judge, and then reach for, a ball on a pedestal. Before reaching, they had to estimate the maximum distance the ball could be away from them to physically reach it, followed by actually reaching for it. Three conditions were varied, involving the hands (one or both), the feet on blocks of varying length, and the support surface being either solid or compliant. It was hypothesized that changes in perceptual demand would differentiate between the children with DCD and typically developing children. This was supported in more than one way. The overall positional variability of the DCD group was greater than that in typically developing children in the highest difficulty condition. Typically developing children reduced their postural motion compared to the low difficulty, whereas the children with DCD did not show such a reduction. As an alternative to visual tasks, a cognitive task was employed and again, a central finding of a reduction in postural motion for both groups was found; further, the reduction between the high and low difficulty was significant for typically developing children, but not for the DCD group. The authors suggest that children with DCD have a diminished perception-action coupling in tasks with high perceptual demands, thus reducing the functional integration of supra-postural task demands and the consequent postural activity. Taken together, these studies suggest that the locus of motor differences between the two groups lies in the link between perception and action, with the biomechanics of the postural system and the perceptual and cognitive elements combining in an embodied context. Typically developing children, compared to children with DCD, appear to be more flexible and adept at naturally adapting to the demands of the environmental context, using all of the personal resources available to them. The unit of analysis is the total childcontext interaction, not solely the child.

Other series of studies have examined ball catching in children with DCD and compared them to typically developing children, with consistent results. Differences between children with DCD and TDC aged 7 to 10 years were examined for balls projected to the midline, to the left shoulder and to the right shoulder, with the requirement of a two handed catch. This task necessitates the child to perceive the environmental demand and make adjustments to these constraints. The children with DCD caught fewer balls and were better at the midline than those projected to either left or right shoulder. The kinematic analysis showed the DCD group showing lower degrees of limb coupling than TDC in the projections to the right and the left, illustrating the influences of task constraints [28]. In a similar vein, again using kinematic analysis with a two handed catch, results showed both groups to evidence coupling of the limbs. However, the
DCD group had less capacity to adapt to the different conditions and this was shown by lower levels of coupling when constraints were increased [29]. The result emphasized that the children with DCD made fewer catches, had a smaller range of motion and had less variable angular excursions in the elbow. The conclusion was that the children with DCD were more rigid and showed less flexibility than the TDC [30].

These studies illustrate the effect that environmental constraints have on the performance of children with DCD on a ball catching task. They not only catch fewer balls, but also are less able to make adjustments to the task constraints shown, either by lower degrees of limb coupling, or by more rigidity in their responses. The children with DCD appear to have a poorer match between their resources and the context, thus invoking an ecological explanation of their difficulties. As the task becomes more difficult, it may require more degrees of freedom which gives difficulties to the children with DCD. Alternatively one could invoke a visual explanation, noting that as the task increases in difficulty, children with DCD are less adept at making fast adaptations through fast dorsal stream channels. To take it a stage further, any attempts at intervention would be to present contextual constraints in a graded and variable fashion, to aid in the attunement of their actions to the differing constraints, such as gradually increasing the difficulty either by speed or location of the ball trajectory.

The preceding studies show perception-action coupling in the visual and kinesthetic domain, but these are not the only modalities where the organism-context transaction occurs. Researchers noted that perception-action coupling had mainly concentrated on vision, but that little had been done with audition [31]. In Whitall's study participants were required to clap while walking in place to an auditory signal. Two groups of children, one DCD and one TDC, both performed poorer than an adult group, but no differences between the two children groups were found in relative phasing, that is how closely individuals timed their foot strike or clap to a metronome beat. However, when variability within trials of relative phasing was examined, there was a difference between the two children's groups, with the DCD being less stable in their coupling. Also both the TDC group and adult group showed four limb coupling patterns which were absent in the DCD group [31]. These different studies examining performance of children with DCD do not simply concentrate on the sensory, cognitive difficulties or invoke such concepts as schemas or motor programs. Instead, all of these are treated as constraints in an ecological system that is self-organizing with multiple components that occur at various levels within a childenvironment-task transaction. These explanations have been commonplace in models for adults, and are becoming stronger in typically developing children with work involving children with DCD promising, but still in its infancy. 


\section{Conclusion}

Two different models of ecological origins have been presented. They have differing origins, often using contrasting methodologies and were aimed at explaining different phenomena. The nested ecological settings of Bronfenbrenner seek to offer explanations of child development over time, and the manner in which these systems interact and influence a child's life. When relating this model to children with DCD, the proposal is that these systems crucially influence the participation of the children, with the obvious subsequent actions being to change some of these systems in order that participation can be enhanced. Participation is essential for competent performance to take place, and the ecological model of Turvey and colleagues offers explanations about the environmental niche, and the fit with the child is the unit of analysis. In the examples shown of reaching and grasping, ball catching, and clapping to an auditory beat, children with DCD show poorer performance and appear to be less attuned to the contextual demands. Both ecological models offer optimism, with the suggestion that if the contextual experiences of children with DCD can be enhanced, this will lead to more appropriate and skillful competence in the general activities of daily living.

\section{Compliance with Ethics Guidelines}

Conflict of Interest David Sugden declares that he has no conflict of interest.

Human and Animal Rights and Informed Consent This article does not contain any studies with human or animal subjects performed by any of the authors.

\section{References}

Papers of particular interest, published recently, have been highlighted as:

- Of importance

.. Of major importance

1.• APA. Diagnostic and statistical manual for mental disorders, 5th revision: DSM-5. Washington DC: American Psychiatric Association; 2013. The most recent edition of the influential diagnostic manual that sets the equivalent procedures world-wide to enable clinicians and researchers to work consistently and reliably.

2. Chen HF, Cohen ES. Social participation for children with developmental coordination disorder: conceptual, evaluation and intervention considerations. Phys Occup Ther Pediatr. 2003;23:61-78.

3. WHO. International classification of functioning, disability and health. Geneva: World Health Organisation; 2001.

4.• Ecological approaches to cognition in sport and exercise. International Journal of Sport Psychology, Special Edition. 2012;40(1):1-226. This important special edition provides excellent summaries, debates about the various ecological positions. It notes their underlying philosophies, methodologies and examines the similarities and difference between various models and applies them to sport and exercise.

5. Bronfenbrenner U. Ecological models of human development, In International Encyclopedia of Education, vol. 3. 2nd ed. Oxford: Elsevier; 1994. p. 1643-7.

6. Bronfenbrenner U, Morris P. The bio-ecological model of human development. In: Damon W, Lerner RM, editors. Handbook of child psychology: Vol. 1. Theoretical models of human development. 6th ed. New York: John Wiley; 2006. p. 793-828.

7. Turvey MT. From physical education to physical intelligence: 50 years of perception-action. AVANT. 2012;III(2):128-38. A personal account of influences on the author's thinking about action and perception and the future of ecological psychology.

8.• Turvey MT, Carello C. On intelligence from first principles: guidelines for inquiry into the hypothesis of physical intelligence (PI). Ecol Psychol. 2012;24(1):3-32. A detailed summary and explanation of the manner in which activity is viewed as a form of intelligence.

9. Gibson JJ. The ecological approach to visual perception. Boston: Houghton Mifflin; 1979.

10. Ericsson KA, Krampe RT, Tesch-Romer C. The role of deliberate practice in the acquisition of expert performance. Psychol Rev. 1993;100:363-406.

11. Driskell JE, Willis RP, Copper C. Effect of overlearning on retention. J Appl Psychol. 1992;77:615-22.

12. Sugden DA, Wade MG. Typical and atypical motor development, Clinics in Developmental Medicine. London: Mackeith Press; 2013. p. 384. A whole text devoted to typical and atypical development and utilizing various ecological models.

13. McConichie H, Colver AF, Forsyth RJ, Jarvis SN, Parkinson KN. Participation of disabled children: how should it be characterised and measured? Disabil Rehabil. 2006;28:1157-64.

14.• Cairney J, Hay JA, Veldhuizen S, Missiuna C, Faught BE. Developmental coordination disorder, gender and the activity deficit over time: a longitudinal analysis. Dev Med Child Neurol. 2010;52(3):e67-72. An examination of if and how participation in children with $D C D$ changes over time.

15.• Liberman L, Ratzon N, Bart O. The profile of performance skills and emotional factors in the context of participation among you children with developmental coordination disorder. Res Dev Disabil. 2013;34(1):87-94. Examines the relationship between participation and hope among children with DCD compared to typically developing children ages 5-6 years.

16.• Kwan MYW, Cairney J, Hay JA, Faugh BE. Understanding physical activity and motivations for children developmental coordination disorder: an investigation using the theory of planned behaviour. Res Dev Disabil. 2013;34(11):3691-8. Through questionnaires and accelerometers this examined examined physical activity and associated cognitions in children with DCD and their typically developing peers.

17.• Sylvestre A, Nadeau L, Charron L, Larose N, Lepage C. Social participation by children with developmental coordination disorder compared to their peers. Disabil Rehabil. 2013;35(21):1814-20. In children aged 5-13 comparisons were made between children with $D C D$ and their typically developing counterparts.on their level of social participation.

18. Dahan-Oliel N, Shikako-Thomas K, Majnemer A. Quality of life and leisure participation in children with neurodevelopmental disabilities: a thematic analysis of the literature. Qual Life Res. 2012;21:427-39. A survey of 19 studies involving quality of life and leisure participation in children with a range of developmental disorders using electronic searches and employing thematic analysis.

19. Cairney J, Rigoli D, Piek J. Developmental Coordination Disorder and internalizing problems in children: the environmental stress 
hypothesis elaborated. Dev Rev. 2013;33(3):224-38. The preceding seven articles are all concerned to a lesser or greater extent with participation in children with DCD or other developmental disorders. They show different aspects of participation ranging from gender differences, to social emotional factors, through to effects upon performance.

20. Sugden DA, Henderson SE. Ecological intervention. London: Pearson; 2007.

21. Law M, Darrah J, Pollack N, King G, Rosenbaum P, Russell D, et al. Family-centred functional therapy for children with cerebral palsy: an emerging, practice model. Phys Occup Ther Pediatr. 1998;18:83-102.

22. Barnett AL, Dawes H, Wilmut K. Constraints and facilitators to participation in physical activities in teenagers with developmental coordination disorder: an exploratory interview study. Child Health Care Dev. 2012;39(3):393-403.

23. Keogh JF, Sugden DA. Movement skill development. New York: McMillan; 1985. p. 384.

24. Newell K. Constraints on the development of coordination. In: Wade MG, Whiting HTA, editors. Motor development in children: aspects of coordination and control. Amsterdam: Martinus Nijhoff; 1986. p. 341-61.

25. Johnson DC, Wade MG. Children at risk for developmental coordination disorder: judgement of changes in action capabilities. Dev Med Child Neurol. 2009;51:397-403.
26. Chen FC, Tsai CL, Stoffregen TA, Chang CH, Wade MG. Postural adaptations to a supra-postural memory task among children with and without developmental coordination disorder. Dev Med Child Neurol. 2011;54:155-9. This study examined post postural adjustments made in response to a supra postural task and the functional integration of these two tasks in children with DCD and their typically developing peers.

27. Chen FC, Tsai CL, Stoffregen TA, Wade MG. Postural responses to a supra-postural visual task among children with and without developmental coordination disorder. Res Dev Disabil. 2011;32: 1948-56. The three preceding articles both examine the effect of environmental task constraints on reaching and grasping in children with DCD.

28. Astill SL. Can children with developmental coordination disorder adapt to task constraints when catching two handed? Disabil Rehabil. 2007;29(1):57-67.

29. Astill SL, Utley A. Two handed catching in children with developmental coordination disorder. Mot Control. 2006;10(2):109-24.

30. Utley A, Steenbergen B, Astill SL. Ball catching in children with developmental coordination disorder: control of degrees of freedom. Dev Med Child Neurol. 2006;49(1):34-8.

31. Whitall J, Getchall N, McMenamin S, Horn C, Wilms-Floet A, Clark J. Perception-action coupling in children with and without DCD: frequency locking between task-relevant auditory signals and motor responses in a dual task. Child Care Health Dev. 2006;32:679-92. 